\title{
El diagnóstico genético del sexo mediante el test de la amelogenina: Métodos y posibles fuentes de error.
}

\author{
Sex typing through the Amelogenin test: Methods and \\ possible pitfalls.
}

\section{F. Francès 1 , A. Castelló ${ }^{2}$ y F. Verdú ${ }^{1}$}

\begin{abstract}
RESUMEN
El diagnóstico del sexo a partir de indicios biológicos es crucial en la ciencia forense en general y en la investigación criminal, en particular. La amelogenina -proteina codificada en los cromosomas sexuales- se viene utilizando con ese fin desde la última década del siglo pasado.

Existen divergencias en secuencia y tamaño entre los alelos codificados en el cromosoma $X$ y el cromosoma Y (AMELX y AMELY, respectivamente). Esta es la base que ha permitido su amplia utilización en ciencias forenses para el diagnóstico genético del sexo. No obstante, recientemente se han publicado casos en los cuales el resultado del test de la amelogenina no corresponde con el sexo legal (oficial) del individuo. El presente trabajo presenta una revisión de los protocolos publicados, localizando las áreas más comúnmente amplificadas del gen de la amelogenina, así como de las técnicas utilizadas para la detección de los fragmentos amplificados de AMELX y AMELY. Por último se analizan las condiciones en las cuales el test de la amelogenina, puede mostrarse discrepante con el sexo fenotípico del individuo y que han de ser tenidas en cuenta para evitar errores potencialmente graves en el curso de la investigación con fines forenses.
\end{abstract}

\begin{abstract}
Sex typing of biological evidences is crucial in forensics in general, and in criminal investigation, in particular. The amelogenin -a protein codified in the sexual chromosomes- has been used with these purposes since the last decade of the past century. There are sequence and size divergences between the $X$ and $Y$-codified alleles of this gene (AMELX and AMELY). This fact is in the base of its using as a genetic sex typing test. However some cases in which the amelogenin test outcome does not correspond with the legal (official) sex have been published.

The present work offers a revision of the published protocols of the amelogenin sex typing test, locating the most common amplified areas of this gene, and the different techniques employed to detect the AMELX and AMELY fragments. Finally, the conditions in which the amelogenin test can differ from the phenotypic sex are analyzed. These conditions must be taken into account in order to avoid potentially serious errors in the forensic investigation.
\end{abstract}

Key words: Amelogenin; Sex Identification; Forensics, Genomics.

Palabras clave: Amelogenina, Identificación del sexo, Forense, Genética.

Correspondencia: Dr. Francesc Francès. Facultad de Medicina, U. D. Medicina Legal, Av. Blasco Ibáñez, ㄲo 15, 46010 Valencia. Teléfono/Fax: +34963864655/+34963864165. E-mail: francesc.frances@uv.es.

1 Doctor en Medicina y Cirugía. Profesor de Medicina Legal y Forense. Universidad de Valencia.

2 Doctora en Ciencias Químicas. Profesora de Medicina Legal y Forense. Universidad de Valencia. 


\section{INTRODUCCIÓN:}

La amelogenina es una proteína presente en el esmalte dental cuyo gen codificante en el ser humano se encuentra en los cromosomas X (Xp22. I-Xp22.3) e Y (Yp I I.2) [I]. La deleción de este gen origina una enfermedad denominada amelogénesis imperfecta ligada al cromosoma $X[2]$.

Existen divergencias entre la secuencia del gen de la amelogenina localizado en el cromosoma X (AMELX) y el localizado en el cromosoma Y (AMELY), debidas tanto a sustituciones puntuales de bases como a inserciones de fragmentos de diferente longitud [I, 3, 4], presentes o ausentes en estos dos alelos (figura I). A pesar de estas diferencias de secuencia, la existencia de zonas que poseen una homología completa permite la amplificación por PCR (Polymerase Chain Reaction) simultánea en ambos alelos de un fragmento mediante un único par de cebadores. Esta posibilidad está en la base de la utilización del test de la amelogenina para el diagnóstico del sexo. La dicha prueba de la amelogenina se ha impuesto como un método rápido, económico y fiable de determinación del sexo, y han aparecido a lo largo del tiempo numerosos protocolos basados en amplificaciones de determinadas regiones de este gen.

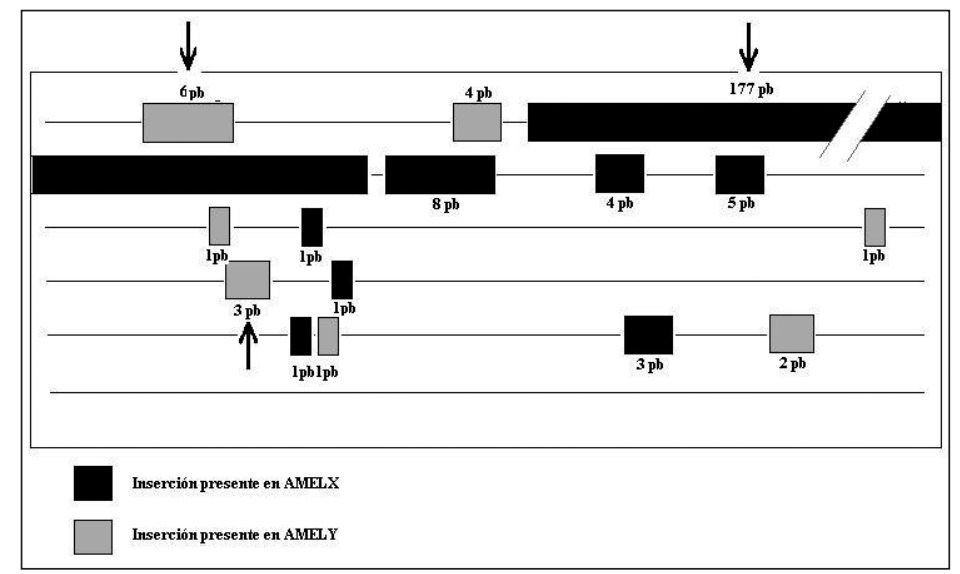

Fig. 1.- Esquema ilustrativo de las diferentes inserciones presentes en los alelos AMELX y AMELY. Se indica el número de bases correspondiente a cada inserción. Las flechas señalan las inserciones más frecuentemente utilizadas para la discriminación de los alelos $X$ e $Y$.

La identificación del sexo a partir de una muestra de ADN obtenida de cualquier fuente biológica (sangre, pelos, saliva, restos óseos, etc), mediante el test de la amelogenina tiene un interés crucial en el ámbito de la medicina forense [5], pero también ha sido propuesta su utilización como medida de control de calidad en estudios epidemiológicos [6], para el diagnóstico prenatal del sexo [7] o en antropología [8].

No obstante, en los últimos años, se han comunicado fallos de esta técnica en determinados individuos, que pueden llevar a un diagnóstico erróneo del sexo, con las consecuencias que este hecho puede tener en la investigación llevada a cabo.

Dada la amplísima utilización de esta técnica en el ámbito forense, nuestro objetivo es presentar una síntesis de los diferentes métodos de diagnóstico del sexo basado en la amplificación de fragmentos del gen de la amelogenina y además, describir las posibles fuentes de error de esta técnica que pueden conducir a atribuciones espurias del sexo correspondiente a una determinada muestra. 
El diagnóstico genético del sexo mediante el test de la amelogenina: Métodos y posibles fuentes de error.

\begin{tabular}{|c|c|c|c|}
\hline CEBADORES & TÉCNICA DETECCIÓN & $\begin{array}{c}\text { TAMAÑO } \\
\text { AMPLICÓN (pb) } \\
\end{array}$ & REFERENCIA \\
\hline 5'-CCCTTTGAAGTGGTACCAGAG-3' & Pirosecuenciación & $x: 45$ & [9] \\
\hline 5'-TGTCTACATACYGGTGGYCTT-3' & & $Y: 48 \mathrm{pb}$ & \\
\hline FAM: 5'-CCTGGGCTCTGTAAAGAATAGTG-3' & Electroforesis capilar & $\mathrm{X}: 96$ & \\
\hline 5'-AGCTTAAACTGGGAAGCTGGTGG-3' & & Y: 102 & [10] \\
\hline 5'-CCC TTT GAA GTGGTA CCAGAG CA -3' & Electroforesis capilar & $x: 80$ & [11] \\
\hline 5'-GCA TGC CTA ATA TTT TCA GGGAAT A-3' & & $Y: 83$ & \\
\hline 5'-ATC CCA GAT GTT TCT CAA GT-3' & Electroforesis capilar & $x: 69$ & [12] \\
\hline 5'-TCA GAG CTT AAA CTG GGA AG-3' & & Y: 75 & \\
\hline 5'-CCCTGGGCTCTGTAAAGAATAGTG-3' & Hibridación (TaqMan) & $x: 106$ & [13] \\
\hline 5'-ATCAGAGCTTAAACTGGGAAGCTG-3' & & $Y: 112$ & \\
\hline \multicolumn{4}{|l|}{ 5'-TATCCCAGATGTTTC-3' AMEX } \\
\hline \multicolumn{4}{|l|}{ 5'-CATCCCAAATAAAGTG-3'AMEY } \\
\hline 5'-CCC TGG GCT CTG TAA AAG AAT CGT-3' & Electroforesis capilar & $x: 106$ & [14] \\
\hline 5'-ATC AGA CTT AAA CTG GGA AGC TG-3' & & Y: 112 & \\
\hline 5'-ACCACCTCATCCTGGGCAC-3 & Electroforesis capilar & $X: 219$ & [15] \\
\hline 5'-ACACAGGCTTGAGGCCAACC-3' & & Y: 225 & \\
\hline 5'-СCCTGGGCTCTGTAAAGAATAGTG-3' & Electroforesis capilar & $x: 104$ & [16] \\
\hline 5'ATCAGAGCTTAAACTGGGAAGCTG-3' & & Y: 110 & \\
\hline 5'-CTCATCCTGGGCACCCTGSTTATATC-3' & AGAROSA & $\mathrm{X}: 1490$ & [17] \\
\hline 5'-GGTACCACTTCARAGGGGTRAGCAC-3' & & $Y: 1310$ & \\
\hline 5'-ACCTCATCCTGGGCACCCTGG-3' & Electroforesis capilar & $x: 212$ & [5] \\
\hline 5'-AGGCCTTGAGGCCAACCATCAG-3' & & Y: 218 & \\
\hline 5'-CTCACCCCTTTGAAGTGGTACC-3' & $\begin{array}{l}\text { Detección productos } \mathrm{PCR} \\
\text { tiempo real }\end{array}$ & $x: 70$ & [18] \\
\hline 5'-TCAGGGAATAAAGAACAAAATGTCTACA-3' & & $Y: 73$ & \\
\hline 5'-CCCTGGGCTCTGTAAAGAATAGTG-3' & DHPLC & $x: 106$ & [19] \\
\hline 5'-ATCAGAGCTTAAACTGGGAAGCTG-3' & & Y: 112 & \\
\hline 5'-CCCCTTTGAAGTGGTACCAG-3' & DHPLC & $x: 81$ & [20] \\
\hline 5'-GCATGCCTAATATTTTCAGGG-3' & & Y: 84 & \\
\hline 5'-CTTGCCTCCTAGCATATAAG-3' & DHPLC & $x: 45$ & [20] \\
\hline 5'-CCATCACACACATTCTTCATC-3' & & $Y: 45$ & \\
\hline 5'-CCCTGGGCTCTGTAAAGAATAGTG-3' & Electroforesis capilar & $\mathrm{X}: 106$ & [21] \\
\hline 5'-ATCAGAGCTTAAACTGGGAAGCTG-3' & & Y: 112 & \\
\hline 5'-CTGATGGTTGGCCTCAAGCCTGTG-3' & AGAROSA & $X: 977$ & [22] \\
\hline 5'-TAAAGAGATTCATTAACTTGACTG-3' & & $Y: 790$ & \\
\hline 5'-CCCTTTGAAGTGGTACCAGAGCA-3' & Electroforesis capilar & $x: 80$ & [23] \\
\hline 5'-GCATGCCTAATATTTTCAGGGAATA-3' & & $Y: 83$ & \\
\hline 5'-CCCTGGGCTCTGTAAAGAAATGTG-3' & AGAROSA & $\mathrm{X}: 122 / \mathrm{Y}: 128$ & $\lceil 24]$ \\
\hline 5'-AGGCTTGAGGCCAACCATCAG-3' & PCR Anidada & $Y: 89$ & \\
\hline 5'-GGGTGGATTCTTCATCCCAAATAA-3' & & & \\
\hline 5'- ATCAGAGCTTAAACTGGGAAGCTG-3' & & & \\
\hline
\end{tabular}

Tabla 1.- Diferentes protocolos de amplificación utilizados para el diagnóstico de género mediante el test de la amelogenina. 


\section{MÉTODOS:}

Amplificación por PCR de fragmentos AMELX y AMELY

Como se ha mencionado anteriormente, debido a la presencia o ausencia de inserciones tanto en AMELY como en AMELX (figura I), han aparecido múltiples protocolos de amplificación de regiones de inserción que generarán fragmentos amplificados de diferente tamaño.

La tabla I muestra diferentes secuencias de cebadores propuestos. Después de una revisión de los diferentes protocolos de amplificación comunicados en la literatura, encontramos tres grandes patrones de diseño de PCR. La mayoría de autores utilizan oligonucleótidos sintetizados para amplificar una región que presenta una inserción de 6 pares de bases (pb) (AAAGTG) en el intrón 3 de AMELY (figura I), no estando estos seis nucleótidos en AMELX. Le siguen en frecuencia las amplificaciones de un fragmento que engloba otra inserción de 3 pb (GAT) (figura I), presente también de forma exclusiva en AMELY. Por último existen protocolos que emplean cebadores que amplifican una amplia región de inserción de 177 pb en AMELX. Estos últimos fragmentos amplificados son grandes (Y790, X 977 e Y I475, XI295 pb, respectivamente) y engloban otras inserciones /deleciones menores que alteran levemente la diferencia final de tamaño de los fragmentos amplificados.

Mención aparte merecen los kits comerciales de identificación como AmpFISTRß (Applied Biosystems, Foster City, USA) y POWERPLEX® (PROMEGA, Madison , USA). Estos preparados permiten determinar un número variable de STRs (Short Tandem Repeats) además de amplificar los fragmentos AMELX y AMELY. La práctica totalidad de ellos emplean protocolos que amplifican el fragmento que engloba la inserción de $6 \mathrm{pb}$ en AMELY mencionada anteriormente.

\section{Detección de fragmentos AMELX y AMELY}

Se han propuesto diversas técnicas para la detección de los fragmentos amplificados específicos $X$ e Y. No obstante, el método más usado en la determinación del sexo mediante el test de la amelogenina es la electroforesis capilar asociada a detección de la fluorescencia procedente del fragmento amplificado. Esto es posible ya que, en la amplificación previa, uno de los cebadores estará marcado con un colorante fluorescente que será detectado a la salida del capilar de electroforesis. Los kits comerciales disponibles, siguen mayoritariamente este patrón de detección de fragmentos AMELX y AMELY.

No obstante, han sido propuestos otros métodos de detección de fragmentos amplificados. Uno de ellos es la electroforesis en geles de agarosa, que, si bien no es una técnica de alto rendimiento, presenta la ventaja de ser económica. Los protocolos diseñados para una posterior electroforesis en agarosa $[22,17]$ requieren diferencias grandes de tamaño ente los fragmentos para su fácil discriminación, y por tanto amplifican regiones que incluyen la gran inserción de 177 pb presente en AMELX (figura I). La figura 2 muestra el resultado de una electroforesis de agarosa a partir del producto de PCR obtenido mediante los cebadores descritos por Eng B et al (22), utilizados usualmente en nuestro laboratorio.

Fig. 2.- Resultado típico del test de la amelogenina tras electroforesis de agarosa a partir de los cebadores propuestos por Eng B et al, 1994 (22). Linea 1: Varón. Línea 2: Mujer. Línea 3: marcador de peso molecular.

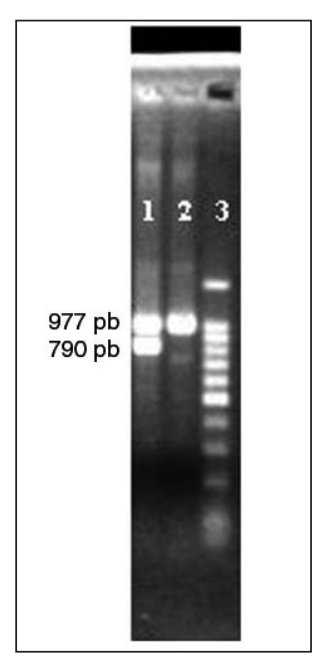


Una técnica alternativa que emplea geles de agarosa es una PCR anidada donde existe una amplificación previa de la zona que incluye la inserción de 6 pb en AMELY y posteriormente una amplificación aleloY-específica sobre la región de la inserción que sólo generará un producto PCR en caso de sexo masculino [24].

Otros métodos menos frecuentemente comunicados son los ensayos de hibridación con sondas fluorescentes como el sistema TaqMan [13] que implican el diseño de sondas específicamente sintetizadas para la hibridación sobre la región de la inserción.

La pirosecuenciación también ha sido utilizada en la detección de AMELX y AMELY. En esta técnica se detecta la secuencia insertada de 3 pb en AMELY [9]. Por último cabe citar modelos de identificación basados en cromatografía líquida de alta resolución desnaturalizante o DHPLC [20], en los cuales generalmente se diferencian fragmentos con inserciones/deleciones, pero destaca un protocolo en concreto de los descritos por Shinka T y colaboradores, en el cual los dos fragmentos amplificados tienen el mismo tamaño, diferenciándose AMELX y AMELY por una única sustitución de una base por otra. Esta es, en cuanto a método, una opción altamente innovadora, ya que no utiliza la clásica discriminación de fragmentos $X$ e $Y$ en función de su tamaño molecular.

Fuentes de discrepancia entre el resultado de la amplificación de fragmentos AMELX y AMELY, y el sexo legal de un individuo

Recientemente han sido comunicados fallos en la tipificación del sexo basada en amplificación de fragmentos del gen de la amelogenina. Además de estas fuentes de error, varias circunstancias adicionales pueden generar resultados en los cuales el sexo genético no coincida con el sexo legal, oficialmente registrado de un individuo.

\section{Deleciones de AMELY}

En 1998, Santos FR y colaboradores comunicaron la existencia en algunos individuos normales de sexo masculino, de deleciones del alelo AMELY, que generaban la ausencia del amplificado correspondiente al sexo masculino, y eran interpretados erróneamente como mujeres [25]. La presencia de deleciones en la región Yp I l.2 del cromosoma Y que afectan el gen de la amelogenina [26] son especialmente frecuentes en población del subcontinente indio $(1,85 \%)$ $[27,28,29]$ siendo más raras en caucásicos $(0,02 \%$ en Austria) (15). Estas deleciones son propias del haplogrupo 12 [28].

\section{Mutaciones en las áreas de hibridación de los cebadores}

Otra causa de error en la tipificación genética del sexo se debe a la presencia descrita de mutaciones en las secuencias de hibridación de los cebadores empleados en el test de la amelogenina [30; 3 I]. En estas condiciones, el cebador no tendrá una homología completa respecto a la secuencia de hibridación en el gen de la amelogenina, y la PCR puede no producir amplificación del fragmento AMELY o AMELX, especialmente si esta discrepancia cebador-patrón se localiza en el extremo 3' del cebador.

\section{Otras causas de discrepancias entre el test de la amelogenina y el sexo legal}

Existen otras circunstancias, ajenas al funcionamiento de la prueba genética de la amelogenina, en las cuales pueden producirse discrepancias entre el resultado de esta prueba y el sexo legal del individuo. 
En caso de mujeres embarazadas de un feto de sexo masculino, la presencia en sangre materna, en determinados periodos de la gestación, de DNA procedente del feto puede generar un resultado masculino del test en estas mujeres gestantes [32, 33]. Tanto es así que se ha propuesto el test de la amelogenina practicado en sangre materna para la detección del sexo del feto [24]. Por otra parte, los transplantes alogénicos de médula ósea, generan una situación de quimerismo, en la cual coexisten células procedentes del donante y del receptor. Esta circunstancia puede generar resultados del test de la amelogenina no coincidentes con el sexo del individuo receptor cuando una mujer recibe un transplante de médula ósea procedente de un donante masculino. De hecho, y aprovechando este fenómeno, se ha utilizado el test de la amelogenina, sola o en combinación con otros STRs como método de control de evolución del injerto de médula ósea $[34,35]$. Además, cabe tener en cuenta que en estas circunstancias, el test de la amelogenina generará resultados discrepantes si la muestra analizada procede de sangre periférica o de raíz pilosa [36].

Por último, únicamente citaremos, ya que no es el propósito de esta revisión, los estados intersexuales, originados bien por diferentes alteraciones cromosómicas relativas a los cromosomas sexuales, bien por anomalías en la respuesta a las hormonas sexuales. Tal es el caso del síndrome de insensibilidad a los andrógenos, la disgenesia gonadal y el déficit de 5 alfa reductasa, además de las situaciones de transexualismo. En todos estos casos es posible la existencia de discrepancias entre el sexo genético y el fenotipo que determina el sexo legal del individuo.

\section{CONCLUSIONES:}

El test de la amelogenina se ha impuesto como una prueba de uso diario en los laboratorios forenses de todo el mundo. Múltiples son los protocolos diseñados con el fin de amplificar unas regiones concretas del gen de la amelogenina y también múltiples son los sistemas de detección, que varían sensiblemente en coste y rendimiento.

No obstante, hemos de tener presente que existen circunstancias en las cuales este test puede generar resultados que no correspondan al sexo fenotípico del individuo. El analista forense ha de tener consciencia de estas circunstancias, lo que permitirá en no pocas ocasiones, una interpretación adecuada de la prueba y podría evitar llegar a conclusiones erróneas con los perjuicios que de ellas se deriven.

\section{BIBLIOGRAFÍA:}

I. Nakahori Y, Takenaka 0, Nakagome Y: A human X-Y homologous region encodes "amelogenin". Genomics 1991; 9:264-269.

2. Lagerström M, Dahl N, Nakahori Y, Nakagome Y, Bäckman B, Landegren U, Pettersson U: A deletion in the amelogenin gene (AMG) causes X-linked amelogenesis imperfecta (AIHI), Genomics 199I, 10:971-975.

3. Lau EC, Mohandas TK, Shapiro LJ, Slavkin HC, Snead ML: Human and mouse amelogenin loci are the sex chromosomes. Genomics. 1989; 4:162-168.

4. Salido EC, Yen PH, Koprivnikar K, Yu LC, Shapiro LJ: The human enamel protein gene amelogenin is expressed from both the $X$ and the $Y$ chromosomes.Am J Hum Genet. 1992; 50:303-316.

5. Sullivan, K.M., Mannucci, A., Kimpton, C.P. and Gill, P: A rapid and quantitative DNA sex test: fluorescence-based PCR analysis of X-Y homologous gene amelogenin. BioTechniques 1993);|15:637-64|.

6.- Francès F, Portolés 0 , González JI, Coltell 0 , Verdú F, Castelló A, Corella D. Amelogenin test: From forensics to quality control in clinical and biochemical genomics. Clin Chim Acta. 2007;386:53-6.

7. Chowdhury MR, Mathur R, Verma IC: Utility of XY-amelogenin gene primers for detection of sex chromosomes.Indian J Med Res. 1998;107:182-186.

8. Faerman M, Filon D, Kahila G, Greenblatt CH, Smith P, Oppenheim A: Sex identification of archaeological human remains based on amplification of the $X$ and $Y$ amelogenin alleles. Gene 1995; 167:327-332.

9. Tschentscher F, Frey UH, Bajanowski T: Amelogenin sex determination by pyrosequencing of short PCR products. Int J Legal Med. 2008; 122:333-335.

10. Wiegand P, Klein R, Braunschweiger G, Hohoff C, Brinkmann B: Short amplicon STR multiplex for stain typing. Int J Legal Med. 2006; 120:160-164. 
II. Liu C, Wang HP, Sun HY, Wang HJ: Establishment of miniCTTA multiplex set with fluorescence-labeled primers. Nan Fang Yi Ke Da Xue Xue Bao. 2006; 26:1574-1576.

12. Bender K, Schneider PM: Validation and casework testing of the BioPlex-II for STR typing of telogen hair roots. Forensic Sci Int. 2006; I61:52-59.

13. Alonso A, Martín P: A real-time PCR protocol to determine the number of amelogenin $(X-Y)$ gene copies from forensic DNA samples. Methods Mol Biol. 2005; 297:31-44.

14. Miścicka-Sliwka D, Grzybowski T, Woźniak M: Optimization of a hexaplex DNA amplification from short tandem repeat and amelogenin loci. Electrophoresis. 1997; 18:1627-1632.

15. Steinlechner M, Berger B, Niederstätter H, Parson W: Rare failures in the amelogenin sex test. Int J Legal Med. 2002; II6:II7-I20. 16. Grubwieser P, Mühlmann R, Berger B, Niederstätter H, Pavlic M, Parson W: A new "miniSTR-multiplex" displaying reduced amplicon lengths for the analysis of degraded DNA. Int J Legal Med. 2006; 120:115-120.

17. Fredsted T, Villessen P.Fast and reliable sexing of prosimian and human DNA. Am J Primatol. 2004; 64:345-350.

18. Roccazzello AM, Tringali G, Barbaro A, Cormaci P, Barbaro A, Insirello E: Simultaneous estimation of a Y-specific fragment, an Xspecific fragment and sex determination of forensic studies in realtime PCR. Forensic Sci Int. 2004;146:165-166.

19. Beraud-Colomb E, Roubin R, Martin J, Maroc N, Gardeisen A, Trabuchet G, Goossens M: Human beta-globin gene polymorphisms characterized in DNA extracted from ancient bones 12000 years old. Am J Hum Genet. 1995;57: 1267-I274.

20. Shinka T, Naroda T, Tamura T, Sasahara K, Nakahori Y: A rapid and simple method for sex identification by heteroduplex analysis, using denaturing high-performance liquid chromatography (DHPLC). J Hum Genet. 2001;46:263-266.

21. Akane A, Shiono H, Matsubara K, Nakahori Y, Seki S, Nagafuchi S, Yamada M, Nakagome Y: Sex identification of forensic specimens by polymerase chain reaction $(P C R)$ : two alternative methods. Forensic Sci Int. 1991; 49:81-88.

22. Eng, B., Ainsworth, P. and Waye, J.S: Anomalous migration of $P C R$ products using nondenaturing polyacrylamide gel electrophoresis: the amelogenin sex-typing system. J. Forensic Sci. 1994; 39:1356-1359.

23. Haas-Rochholz H, Weiler G: Additional primer sets for an amelogenin gene PCR-based DNA-sex test. Int J Legal Med. 1997;110:312-315.
24. Zhu B, Sun QW, Lu YC, Sun MM, Wang LJ, Huang XH: Prenatal fetal sex diagnosis by detecting amelogenin gene in maternal plasma. Prenat Diagn. 2005; 25:577-58I.

25. Santos FR, Pandya A, Tyler-Smith C: Reliability of DNA-based sex tests. Nat Genet. 1998; 18:103.

26. Lattanzi W, Di Giacomo MC, Lenato GM, Chimienti G, Voglino G, Resta N, Pepe G, Guanti G: A large interstitial deletion encompassing the amelogenin gene on the short arm of the $Y$ chromosome. Hum Genet. 2005; II6:395-40I.

27. Thangaraj K, Reddy AG, Singh L: Is the amelogenin gene reliable for gender identification in forensic casework and prenatal diagnosis? Int J Legal Med. 2002; II6:I2I-I23.

28. Chang YM, Burgoyne LA, Both K: Higher failures of amelogenin sex test in an Indian population group. J Forensic Sci. 2003; 48:1309-1313.

29. Kashyap VK, Sahoo S, Sitalaximi T, Trivedi R: Deletions in the Yderived amelogenin gene fragment in the Indian population. BMC Med Genet. 2006; 7:37.

30. Roffey PE, Eckhoff Cl, Kuhl JL: A rare mutation in the amelogenin gene and its potential investigative ramifications. J Forensic Sci. 2000; 45:1016-1019.

3I. Shadrach B, Commane M, Hren C, Warshawsky I: A rare mutation in the primer binding region of the amelogenin gene can interfere with gender identification. J Mol Diagn. 2004; 6:401-405.

32. Bianchi DW: Circulating fetal DNA: Its origin and diagnostic potential. Placenta 2004; 25:93-101.

33. Lo YM, Corbetta N, Chamberlain PF et al : Presence of fetal DNA in maternal plasma and serum. Lancet. 1997; 350:485-487.

34. Ghaffari SH, Chahardouli B, Gavamzadeh A, Alimoghaddam K: Evaluation of hematopoietic chimerism following allogeneic peripheral blood stem cell transplantation with amelogenin marker. Arch Iran Med. 2008; 11:35-41.

35. Sufliarska S, Minarik G, Horakova J, Bodova I, Bojtarova E, Czako B, Mistrik M, Drgona L, Demitrovicova M, Lakota J, Krivosikova M, Kovacs L: Establishing the method of chimerism monitoring after allogeneic stem cell transplantation using multiplex polymerase chain reaction amplification of short tandem repeat markers and Amelogenin. Neoplasma. 2007; 54:424-430.

36. von Wurmb-Schwark N, Bosinski H, Ritz-Timme S: What do the $X$ and $Y$ chromosomes tell us about sex and gender in forensic case analysis? J Forensic Leg Med. 2007; 14:27-30. 